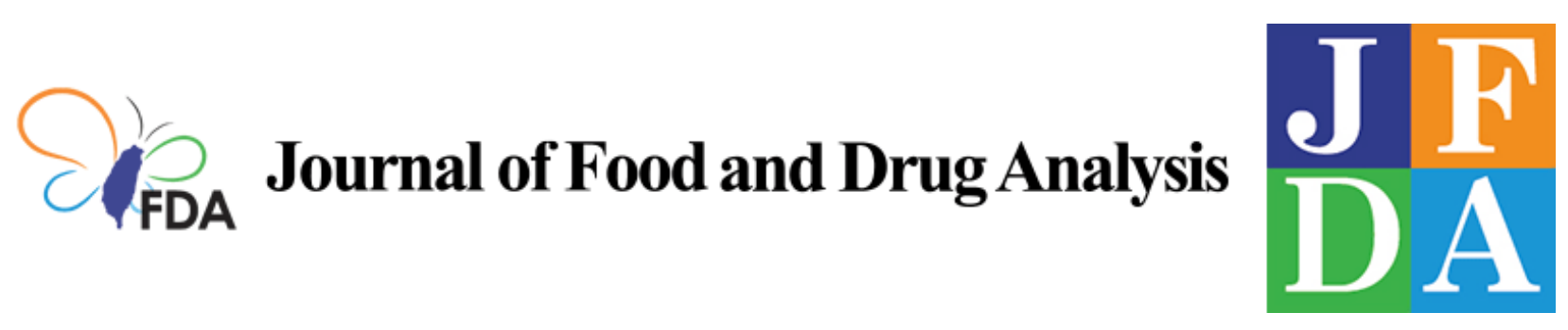

\title{
Quantification of Adenosine Mono-, Di- and Triphosphate from Royal Jelly using Liquid Chromatography - Tandem Mass Spectrometry
}

Follow this and additional works at: https://www.jfda-online.com/journal

Part of the Food Science Commons, Medicinal Chemistry and Pharmaceutics Commons, Pharmacology Commons, and the Toxicology Commons

(c) (i) (9)

This work is licensed under a Creative Commons Attribution-Noncommercial-No Derivative Works 4.0 License.

\section{Recommended Citation}

Liao, Wan-Rou; Huang, Jen-Pang; and Chen, Sung-Fang (2020) "Quantification of Adenosine Mono-, Diand Triphosphate from Royal Jelly using Liquid Chromatography - Tandem Mass Spectrometry," Journal of Food and Drug Analysis: Vol. 28 : Iss. 3 , Article 2.

Available at: https://doi.org/10.38212/2224-6614.1007

This Original Article is brought to you for free and open access by Journal of Food and Drug Analysis. It has been accepted for inclusion in Journal of Food and Drug Analysis by an authorized editor of Journal of Food and Drug Analysis. 


\title{
Quantification of adenosine Mono-, Di- and triphosphate from royal jelly using liquid chromatography - Tandem mass spectrometry
}

\author{
Wan-Rou Liao ${ }^{a}$, Jen-Pang Huang ${ }^{b}$, Sung-Fang Chen ${ }^{a, *}$ \\ ${ }^{a}$ Department of Chemistry, National Taiwan Normal University, Taipei, Taiwan \\ ${ }^{\mathrm{b}}$ MSonline Scientific Co., Ltd., Taipei, Taiwan
}

\begin{abstract}
Nucleotides are composed of nitrogen bases, ribose units and phosphate groups. Adenine (Ade), adenosine monophosphate (AMP), adenosine diphosphate (ADP) and adenosine triphosphate (ATP) all play important roles in physiological metabolism. Royal jelly, a secretion produced by worker bees, contains a variety of natural ingredients and several studies have shown that royal jelly can serve as a source of nutrition for humans. In this study, a rapid and effective LC/ MS method coupled with pre-processing methods was developed and validated for the accurate quantification of Ade, AMP, ADP and ATP in royal jelly. To achieve the best extraction efficiency, two pretreatment methods, namely, solidphase extraction (SPE) and dispersive solid-phase extraction (dSPE), were developed and investigated. Silica-based cyanopropyl $(\mathrm{CN})$ liquid chromatography was employed using $\mathrm{pH}$ programming with a quaternary mobile phase system for the analyses. The total LC/MS run time was less than $12 \mathrm{~min}$ with a constant flow rate of $0.25 \mathrm{~mL} / \mathrm{min}$. The linear range were $2.5-1000 \mathrm{ng} / \mathrm{mL}$ with a correlation coefficient $\mathrm{r}=0.9995$. The limit of detection (LOD) of Ade, AMP, ADP and ATP was 1, 1, 2.5 and $5 \mathrm{ng} / \mathrm{mL}$; the limit of quantitation (LOQ) was 2.5, 2.5, 5 and $10 \mathrm{ng} / \mathrm{mL}$, respectively. Precision (RSD\% $<10.5 \%$ ) and accuracy (recovery $81.3-118.4 \%$ ) were satisfactory for both two pre-processing methods. Nucleotides were successfully quantified from 2-day and 3-day royal jelly samples, with concentrations within $6.2-2126.0 \mathrm{mg} / \mathrm{kg}$.
\end{abstract}

Keywords: dSPE, HPLC-MS/MS, Nucleotide, Royal jelly, SPE

\section{Introduction}

$\mathrm{N}$ ucleotides, which are composed of a nitrogen base, ribose and a phosphate group(s) such as adenosine $5^{\prime}$-monophosphate (AMP), adenosine $5^{\prime}$-diphosphate (ADP) and adenosine $5^{\prime}$-triphosphate (ATP) all play important roles in physiological metabolism [1]. Nucleotides, nucleosides and nucleobases are the basic components of all cells, forming DNA and RNA that contain and transmit genetic information in a cell. The conversion of ATP to ADP and AMP in cells produces energy that permit a variety of physiological functions to occur and as a result, ATP is known as intracellular energy currency [2].

Any food composed of living cells including animal viscera, seafood, fresh fruits, vegetables and mushrooms contain nucleosides and nucleotides $[3,4]$, which are essential nearly for all biological processes. Nucleotides and nucleoside can enter the human body through digested food, then be absorbed and utilized. For example, infant formulas are frequently supplemented with AMP. The freshness of seafood also uses AMP, ADP and ATP levels as an index.

Royal Jelly, a pale yellow viscous jelly-like substance, is secreted by mandibular glands and hypopharyngeal glands of worker bees after collecting and drinking pollen or nectar. Fresh royal jelly is composed of $50-60 \%$ water, $18 \%$ protein, $15 \%$ hydrocarbon, $3-6 \%$ lipid, $1.5 \%$ minerals, trace amounts of vitamins and AMP, ADP, ATP [5-9]. Although royal jelly is a secretion that does not contain cells, it is also rich in adenine and its corresponded nucleotides and can be regarded as a health food. Royal Jelly combines anti-aging, anti-

Received 30 January 2020; accepted 27 April 2020.

Available online 28 August 2020.

* Corresponding author at: Department of Chemistry, National Taiwan Normal University, Taipei, Taiwan

E-mail address: sfchen@ntnu.edu.tw (S.-F. Chen). 
inflammatory, anti-tumor, anti-bacterial, antiallergic, anti-oxidant properties and other health benefits, and functions to improve metabolism, enhance immunity and improve physical fitness in the body [10-15]. Commercially available fresh royal jelly is harvested by a beekeeper in a queen cell cup and is denoted as two-day-old and threeday-old queen bee material according to the time of harvest.

Due to the high polarity of nucleosides and nucleotides, solid-liquid extraction, using a polar solvent is frequently used as an extraction method. However, royal jelly contains proteins and lipids that can create severe matrix effects which make the analysis difficult. Most studies dealing with the extraction of royal jelly have used the solid-liquid extraction with an ultrasonic processor or vortex $[16,17]$. A number of methods have been developed for the detection and quantitation of nucleotides and analogs thereof. Because of its selectivity, high sensitivity, and rapid response time, a label-free fluorescent ATP sensor was developed for the detection and quantification of nucleotides [18]. Other analytical methods, including capillary electrophoresis (CE) have also been developed [19-21]. However, these methods all have certain drawbacks regarding their reproducibility and detection limits.

Liquid chromatography - tandem mass spectrometry (LC-MS/MS), ion-pair reversed-phase liquid chromatography and reverse phase liquid chromatography-UV are currently gaining in popularity and have become reliable techniques for the analysis of nucleosides and nucleotides [22-24]. For such a methodology to be used, selecting a suitable stationary phase that permits nucleosides and nucleotides to be individually retained without severe peak tailing, which is commonly observed in conventional C18-based chromatography, is a critical issue. Octadecylsilane (ODS, C18) [17,25,26], HILIC and ion-exchange columns are currently widely used in the analysis of nucleosides and nucleotides [27-30]. A newly developed method utilizing porous graphitic carbon (PGC) columns and a quaternary mobile phase system that incorporates acidic properties was also reported [31].

In this study, SLE-SPE and SLE-dSPE pre-processing methods were developed for the efficient and improved extraction of adenine, AMP, ADP and ATP from royal jelly. In the case of SLE, different types of extraction solvents, extraction reagents and ultrasonic extraction times were investigated. The adsorbent efficiency of dSPE sorbents was also investigated via the combined use of several sorbents. Combining with this an efficient sample pretreatment, a cyanopropyl $(-\mathrm{CN})-$ based LC-MS method was also developed for the separation and quantitation of adenine and its corresponded nucleotides.

\section{Material and methods}

\subsection{Standard and reagents}

Analytical standards of adenine (Ade), adenosine $5^{\prime}$-diphosphate (ADP) and adenosine 5'-triphosphate (ATP) were obtained from Bio Basic Inc. (Toronto, Canada); adenosine 5'-monophosphate (AMP) was obtained from MP Biomedicals ${ }^{\mathrm{TM}}$, (State, USA). Isotope-labelled internal standard $A M P^{15} \mathrm{~N}_{5}$ was purchased from Sigma-Aldrich, (St., USA).

Ultrapure water (Milli-Q) was produced by a Millipore system (France). Acetic acid and citric acid were obtained from Sigma-Aldrich, St. Louis, USA. Ammonia water $(28 \%)$ was acquired from Nihon Shiyaku Reagent, Japan. The three kinds of sorbents used in dispersive solid phase extraction (dSPE) step are listed below: Primary and secondary amine (PSA) SPE bulk sorbent (Agilent Technologies, USA), C18 Endcapped SPE bulk sorbent (C18EC), $57.9 \mu \mathrm{m}, 59$ A (Agilent Technologies, USA), Graphitized Carbon Black (GCB) (Waters, Ireland). The Oasis ${ }^{\circledR}$ MAX Cartridge used in solid phase extraction (SPE), $30 \mu \mathrm{m}$ (Waters, USA).

\subsection{Sample}

Royal jelly was harvested from a local apiary in Nantou, Taiwan. Two types of royal jelly were categorized as 2-day royal jelly and 3-day royal jelly based on time after harvest.

\subsection{Sample preparation}

\subsection{1. dSPE sample preparation}

All royal jelly (RJ) was collected from a local apiary and stored at $-20{ }^{\circ} \mathrm{C}$. In a typical run, $1.0 \mathrm{~g}$ of $\mathrm{RJ}$ was accurately weighed into a $50 \mathrm{~mL}$ tube, and $10 \mathrm{~mL}$ of a $20 \%$ aqueous $\mathrm{MeOH}$ extraction solution was added. The mixture was vortexed for $3 \mathrm{~min}$ at temperature, then centrifuged at $8000 \mathrm{rpm}$ at $10{ }^{\circ} \mathrm{C}$ for $10 \mathrm{~min}$; and $2 \mathrm{~mL}$ of the upper layer (organic layer) was transferred to a second $15 \mathrm{~mL}$ tube. In the dSPE step, $300 \mathrm{mg}$ C18EC was added to the $15 \mathrm{~mL}$ tube. This tube was vortexed for $1 \mathrm{~min}$, and centrifuged at $8000 \mathrm{rpm}$ at $10^{\circ} \mathrm{C}$ for $30 \mathrm{~min}$. Next, $300 \mu \mathrm{L}$ of the liquid extract was transferred to a $1.5 \mathrm{~mL}$ glass vial containing $600 \mu \mathrm{L} \mathrm{H}_{2} \mathrm{O}$ and used for the analysis. 


\subsubsection{SPE sample preparation}

A total of $1.0 \mathrm{~g}$ of $\mathrm{RJ}$ was weighed into a $50 \mathrm{~mL}$ tube, and $10 \mathrm{~mL}$ of a $20 \%$ aqueous $\mathrm{MeOH}$ extraction solution was then added. The mixture was vortexed for $3 \mathrm{~min}$ at room temperature, then centrifuged at $8000 \mathrm{rpm}$ at $10^{\circ} \mathrm{C}$ for $10 \mathrm{~min}$; and $2 \mathrm{~mL}$ of the upper layer (organic layer) was transferred to a second $2 \mathrm{~mL}$ Eppendorf tube as the sample solution. In the SPE step, the supernatant was passed through an Oasis $^{\circledR}$ MAX cartridge, that had previously been activated with $1 \mathrm{~mL} \mathrm{MeOH}$ and $1 \mathrm{~mL}$ of $\mathrm{H}_{2} \mathrm{O}$. A $2 \mathrm{~mL}$ aliquot of the upper layer sample solution was added. Washing was achieved with $2 \mathrm{~mL} 1 \%$ $\mathrm{NH}_{4} \mathrm{OH}$ in $\mathrm{ACN}$, and elution was achieved with $2 \mathrm{~mL}$ a $2 \%$ acetic acid. Next, $300 \mu \mathrm{L}$ of the homogeneous elution solution was collected in to a $1.5 \mathrm{~mL}$ glass vial containing $600 \mu \mathrm{L} \mathrm{H}_{2} \mathrm{O}$ and was used for analysis.

\subsubsection{Quantification of Ade, AMP, ADP and ATP}

In this study, the quantification of Ade, AMP, ADP and ATP was performed using the standard addition method due to lack of royal jelly blank matrix. The extract was 10-fold diluted for direct LC-MS analysis. One hundred $\mu \mathrm{L}$ of the homogeneous elution solution was collected in to a $1.5 \mathrm{~mL}$ glass vial containing $100 \mu \mathrm{L} 1000$ ppb $\mathrm{AMP}^{15} \mathrm{~N}_{5}, 100 \mu \mathrm{L} 10$ fold mixed-standard and $700 \mu \mathrm{L} \mathrm{H}_{2} \mathrm{O}$ for the analysis.

\subsection{Preparation of standard solutions}

Individual standard stock solutions $(1.0 \mathrm{mg} / \mathrm{mL})$ were prepared by dissolving $1 \mathrm{mg}$ of the respective compounds in $1 \mathrm{~mL}$ of $2 \mathrm{mM} \mathrm{NaH} \mathrm{PO}_{4}$ to maintain the stability of the sample. A mixed standard working solution $(100 \mu \mathrm{g} / \mathrm{mL})$ was then diluted with Milli-Q water to prepare a series of concentrations of $0.25,0.5,1,2.5,5,10,25,50,100,250,500$ and $1000 \mathrm{ng} / \mathrm{mL}$. Both stock and working solutions were freshly prepared before each experiment.

\subsection{LC/MS analysis}

HPLC analyses were performed on an Agilent 1100 HPLC system (Agilent, USA) with a Shim-pack GIS CN column, $150 \times 2.1 \mathrm{~mm}, 3 \mu \mathrm{m}, 100$ A (Shimadzu, Japan). The column temperature was maintained at $40{ }^{\circ} \mathrm{C}$. The composition of mobile phase A was $\mathrm{H}_{2} \mathrm{O}$, mobile phase $\mathrm{B}$ was $0.5 \%$ Acetic acid, and mobile phase $C$ was $0.1 \%$ Ammonium hydroxide, and mobile phase D was $20 \mathrm{mM}$ Citric acid. The linear gradient flow program: $0-0.3 \mathrm{~min}$, $82.5 \% \mathrm{~A}, 10 \% \mathrm{~B}, 2.5 \% \mathrm{C}, 5 \% \mathrm{D} ; 0.3-0.4 \mathrm{~min}, 87.4 \% \mathrm{~A}$, $10 \% \mathrm{~B}, 2.5 \% \mathrm{C}, 0.1 \% \mathrm{D} ; 0.4-1 \mathrm{~min}$, increasing linearly to $87.4 \% \quad \mathrm{~A}, 10 \% \quad \mathrm{~B}, 2.5 \% \quad \mathrm{C}, \quad 0.1 \% \quad \mathrm{D}$; $1-2.5 \mathrm{~min}, 59.9 \% \mathrm{~A}, 10 \% \mathrm{~B}, 30 \% \mathrm{C}, 0.1 \% \mathrm{D}$; 2.5-3.5 min, increasing linearly to $59.9 \% \mathrm{~A}, 10 \% \mathrm{~B}$, $30 \% \mathrm{C}, 0.1 \% \mathrm{D} ; 3.5-4.5 \mathrm{~min}, 87.4 \% \mathrm{~A}, 10 \% \mathrm{~B}, 2.5 \% \mathrm{C}$, $0.1 \% \mathrm{D} ; 4.5-10 \mathrm{~min}$, increasing linearly to $87.4 \% \mathrm{~A}$, $10 \% \mathrm{~B}, 2.5 \% \mathrm{C}, 0.1 \% \mathrm{D} ; 10-10.01 \mathrm{~min}, 82.5 \% \mathrm{~A}, 10 \%$ $\mathrm{B}, 2.5 \% \mathrm{C}, 5 \% \mathrm{D}$. The total run time was $12 \mathrm{~min}$ with a constant flow rate of $0.25 \mathrm{~mL} / \mathrm{min}$. Chromatograms of all analytes and internal standards in the solvent are shown in Fig. 1.

Mass spectrometry was performed on a AB Sciex API 4000 triple quadrupole mass spectrometry (Applied Biosystems/MDS Sciex, Canada) with a Turbo $\mathrm{V}$ ion source equipped with electrospray ionization (ESI). The ESI settings were operated in the negative ion mode, ion spray voltage at $-4500 \mathrm{~V}$. Instrumental parameters were set as follows: curtain gas at 10 psi, gas 1 and gas 2 at 50, desolvation temperature at $650{ }^{\circ} \mathrm{C}$, multiple reaction monitoring (MRM) transitions and the optimal voltage parameters for nucleotides are given in Supplementary material Table S1. Data were collected using the Analyst $^{\circledR}$ 1.6.2 (AB Sciex).

\subsection{Method validation}

Calibration curves were constructed using peak areas obtained using the standards. Each calibration curve was performed in triplicate with at least seven-point concentrations. The square of the correlation coefficients and linear equation of the calibration curves were used to define linearity. The linear relationship $y=a x+b$, where $x$ is the concentration of the standard solution $(\mathrm{ng} / \mathrm{mL})$ and $\mathrm{y}$ is the peak area of the standard solution divided by the peak area of the $100 \mathrm{ng} / \mathrm{mL}$ isotope-labelled internal standard solution was obtained. The LOD and LOQ were determined based on signal-to-noise ratios of 3 and 10, respectively.

\section{Results and discussion}

\subsection{Optimization of solid-liquid extraction conditions}

In order to obtain a satisfactory extraction efficiency, an efficient pre-processing procedure for removing interfering matrix constituents which influence the analysis is critical. Among these preprocessing steps, the extraction reagent, extraction volume and ultrasonic extraction time were evaluated.

To compare their extraction efficiency, different amounts of extraction solvent $(10 \mathrm{~mL}, 15 \mathrm{~mL}$ and $20 \mathrm{~mL})$, extraction reagent $\left(\mathrm{H}_{2} \mathrm{O}, 20 \% \mathrm{MeOH}, 20 \%\right.$ 


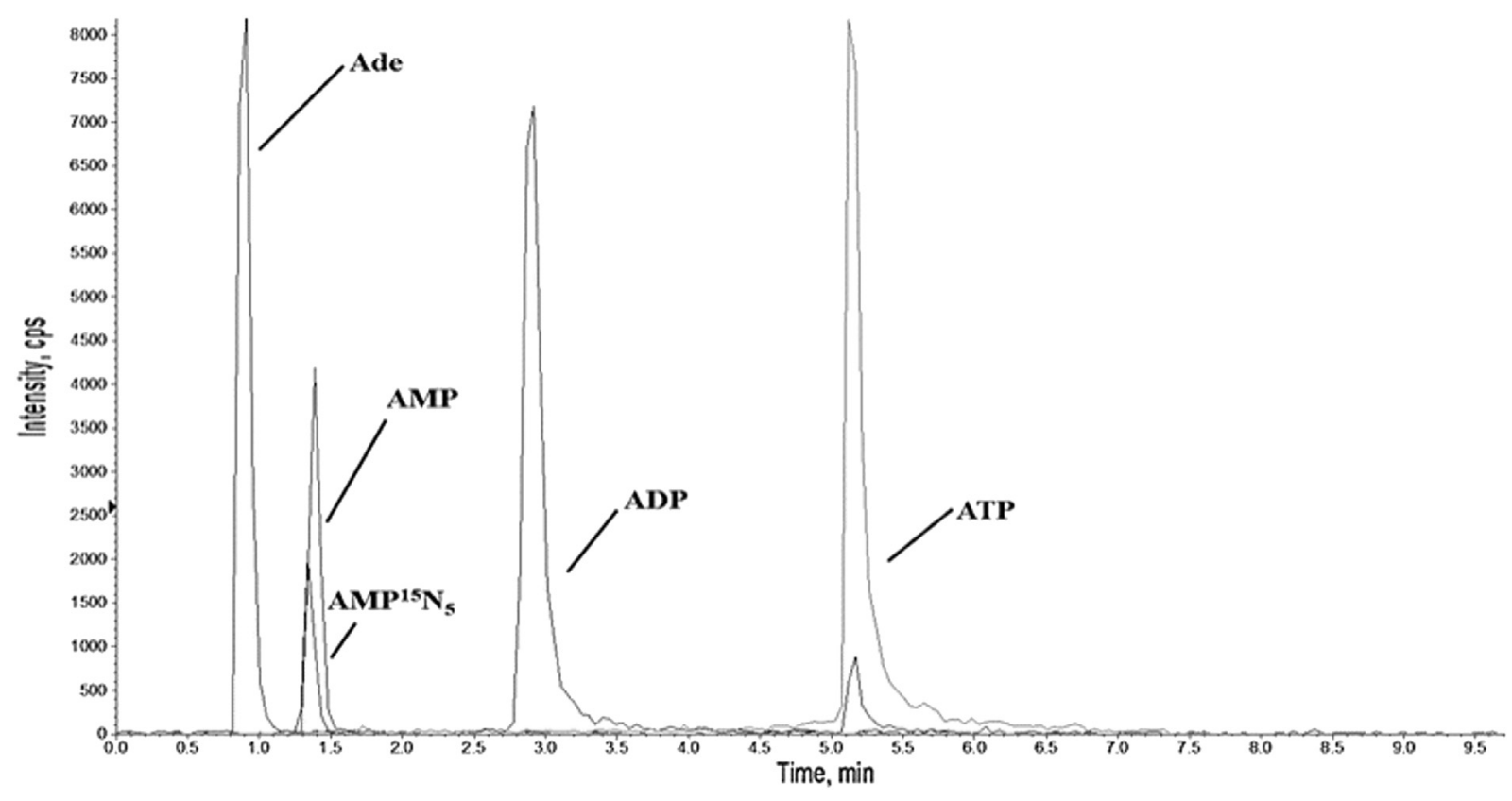

Fig. 1. MRM spectra of 100 ppb of ATP, ADP, AMP, Ade and $A M P{ }^{15} N_{5}$ (isotope-labeled internal standard) mixed-standard solution.

$\mathrm{ACN}, 50 \% \mathrm{MeOH}$ and $50 \% \mathrm{ACN})$ and ultrasonic extraction time ( $0 \mathrm{~min}, 5 \mathrm{~min}, 10 \mathrm{~min}$ and $15 \mathrm{~min}$ ) were investigated and the results are shown in Fig. 2 (A) (B) (C). Overall, $20 \% \mathrm{MeOH}$ is a good extraction solvent for the target analytes and suitable for following dSPE method which using dispersive EC-
C18 sorbent in solution for matrix removal. The results indicated that the best extraction efficiency involved the use of $10 \mathrm{~mL}$ of $20 \%$ aqueous $\mathrm{MeOH}$ as the extraction solvent without ultrasonic extraction for the Ade, AMP, ADP and ATP in royal jelly.
(A)

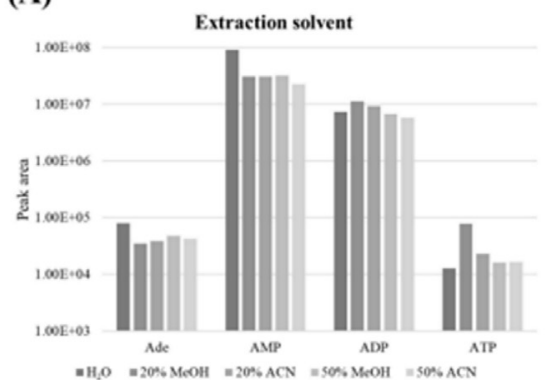

(D)

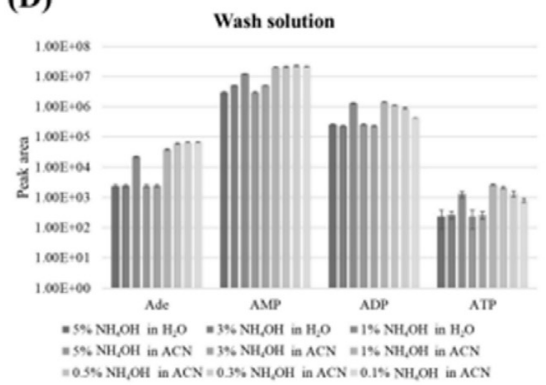

(B)

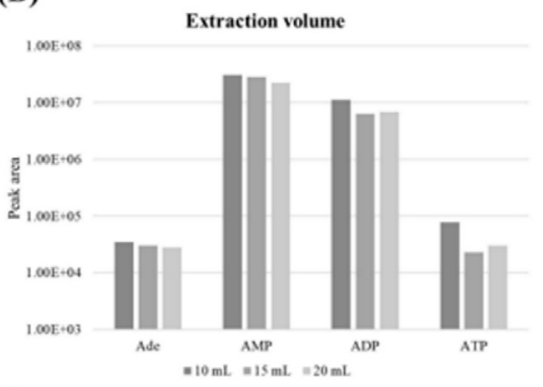

(E)

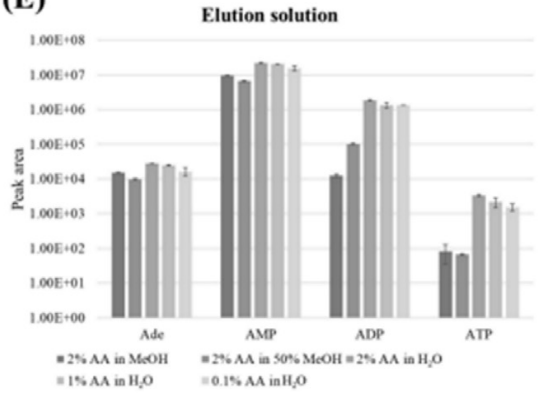

(C)

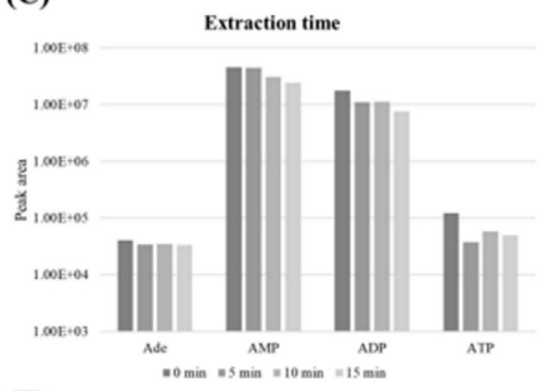

(F)

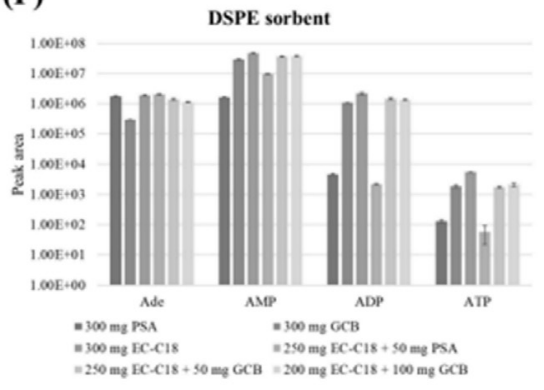

Fig. 2. Comparisons of peak areas of analytes by optimizing pre-processing parameters using royal jelly as the sample. Influence of different (A) extraction solvents, (B) extraction volumes and (C) ultrasonic extraction times in the SLE method. Influence of different (D) wash solutions and (E) elution solutions in the SPE method. Influence of different $(F)$ sorbents in the dSPE method. 


\subsection{Optimization of SPE and dSPE conditions}

\subsubsection{Optimization of solid-phase extraction (SPE)}

Nucleotides are water soluble and negatively charged in a weakly acidic to a basic environment. Oasis MAX, a mixed-mode anion exchange reverse phase column, is the preferred cartridge for solidphase extraction. In order to effectively reduce matrix effects, different wash solution conditions $\left(1-5 \% \mathrm{NH}_{4} \mathrm{OH}, 0.1 \%-5 \% \mathrm{NH}_{4} \mathrm{OH}\right.$ in $\left.\mathrm{ACN}\right)$ and elution solutions (0.1-2\% Acetic acid, 2\% Acetic acid in $\mathrm{MeOH}, 2 \%$ Acetic acid in $50 \% \mathrm{MeOH}$ ) were evaluated.

As shown in Fig. 2 (D) (E), in the case of the wash solution, no significant differences were found between $A C N$ and $\mathrm{H}_{2} \mathrm{O}$, but when $\mathrm{ACN}$ was used as a wash solution the protein matrix precipitated, an ideal occurrence for a mass spectrometry analysis. Comparing different concentrations of $\mathrm{NH}_{4} \mathrm{OH}$, when a high concentration of $\mathrm{NH}_{4} \mathrm{OH}$ was added, the positive charge of the SPE MAX sorbent was weakened, and negatively charged nucleotide analytes were not efficiently retained by MAX sorbent; when a low concentration of $\mathrm{NH}_{4} \mathrm{OH}$ was added, the nucleotides acquired a strong negative charge and were effectively captured by the MAX sorbent. The eluting solution used was $2 \%$ acetic acid, which permitted the nucleotides to be efficiently eluted from the MAX sorbent, especially ADP and ATP. It is because hydrophilic nucleotides are more stable in $\mathrm{H}_{2} \mathrm{O}$, not in high content of organic solvent. In addition, the sample was suitable for connecting the LC analysis using aqueous mobile phase. Based on the above results, $1 \% \mathrm{NH}_{4} \mathrm{OH}$ in $\mathrm{ACN}$ and $2 \%$ acetic acid was used, respectively, as the washing and eluting solutions for solid phase extraction.

\subsubsection{Optimization of dispersive solid-phase extraction (dSPE)}

In order to reduce matrix effects, it is imperative to use a sorbent that can effectively remove the adsorbed matrix from the royal jelly samples. There are many types of commercially available dSPE sorbents with different characteristics that are suitable for use as analytes. The adsorbent efficiency of the dSPE sorbents was evaluated for a combination of different sorbents (300 mg PSA, $300 \mathrm{mg}$ GCB, $300 \mathrm{mg}$ EC-C18, $250 \mathrm{mg}$ EC-C18 + $50 \mathrm{mg}$ PSA, $250 \mathrm{mg}$ EC-C18 + $50 \mathrm{mg}$ GCB and $200 \mathrm{mg}$ EC$\mathrm{C} 18+100 \mathrm{mg}$ GCB). Using PSA as a sorbent can remove polar compounds including polar pigments, sugars and fatty acids; Graphitized carbon black, $\mathrm{GCB}$, as a sorbent can remove pigment and sterols; End-Cap C18, EC-C18, as a sorbent can remove non-polar compound including lipids.
As shown in Fig. 2, Nucleotide compounds were retained by the PSA and GCB sorbent. However, the use of EC-C18 as a dSPE sorbent not only removed non-polar species, but also permitted high-polarity analytes to be retained resulting in a satisfactory purification.

Based on the above findings, $300 \mathrm{mg}$ EC-C18 was selected as the best sorbent for dispersive solid phase extraction.

\subsection{Chromatographic separation and MS/MS parameters}

Phosphate buffer has long been used for the separation of nucleic acids on UV detection system $[16,32]$. Although it provides a fairly satisfactory LC resolution, there is still a void if electrospray mass spectrometry is favored for superior sensitivity, since phosphate buffer is not a preferred choice when using with ESI. In this study, a cyanopropyl $(\mathrm{CN}-)$ column with the $\mathrm{pH}$ of the quaternary mobile phases being programmed was employed for the separation and quantitative analyses of the target analytes. The cyanopropyl (CN-) group has polar properties and can also serve as a stationary phase in either normal or reverse phase chromatography. As a result, it is also capable of retaining negatively charged analytes by regulating the $\mathrm{pH}$ of the eluent. The retention and selectivity of analytes in cyanopropyl-based chromatography can be adjusted by programming the $\mathrm{pH}$ of the mobile phases. In weakly acidic conditions, the cyanopropyl $(\mathrm{CN}-)$ group acquires relative positive charge characteristics that permit negatively charged nucleotides to be attracted and retained. However, when the alkalinity of the mobile phase is increased, the magnitude of the ion pair interactions between cyanopropyl groups and the nucleotides becomes weaker, thus permitting all compounds Ade, AMP, ADP and ATP to be sequentially eluted from the cyanopropyl column. Furthermore, they also help to stabilize the equilibrium in ion pair chromatography.

In this study, the concentrations of four mobile phases including $\mathrm{H}_{2} \mathrm{O}, 0.5 \%$ acetic acid, $0.04 \%$ ammonium hydroxide and $20 \mathrm{mM}$ citric acid were adjusted so as to control the $\mathrm{pH}$ of the eluent and to maintain the stability of the LC system. Acetic acid and aqueous ammonia are frequently used to manipulate the $\mathrm{pH}$ of a mobile phase in LC/MS analyses. By varying the composition of acetic acid, aqueous ammonia and the presence of an ammonium acetate salt, the appropriate retention and separation of Ade, AMP, ADP and ATP can be successfully achieved. Nucleotides such as ADP and 
ATP have a strong affinity for the metal in the injector needle, the injection valve and transporting components of typical HPLC systems. This situation can be eliminated by using citric acid. The use of citric acid also avoids carryover, which also improves the sensitivity of the overall analysis.

The multiple reaction monitoring (MRM) scan mode was used for nucleotide quantitation in samples obtained from cell lysates. Furthermore, four transmission voltages, including DP (declustering potential), EP (entrance potential), CE (collision energy), CXP (collision cell exit potential) for the MRM transitions were optimized. Each MRM transition and the mass spectrometry parameters are shown in Table S1.

\subsection{Method validation}

\subsubsection{Calibration curves and limits of detection (LOD) and quantification (LOQ)}

As shown in Table 1, the calibration curve was constructed using at least seven-point concentrations in triplicate. A linear range between 2.5 and $1000 \mathrm{ng} / \mathrm{mL}$ with a high correlation coefficient $(r>0.9995)$ was found. The limit of detection (LOD) and limit of quantification (LOQ) were determined to be $1-5 \mathrm{ng} / \mathrm{mL}$ and $2.5-10 \mathrm{ng} / \mathrm{mL}$, respectively. The overall recovery ranged from 81.3 to $118.4 \%$, with RSD $<10.5 \%$.

\subsubsection{Accuracy and precision}

Validation of this method involved determining the Intra-day and Inter-day precision and recovery at low-, medium-, high-concentrations $(0.5,1,5 \mu \mathrm{g} /$ $\mathrm{mL}$ ) of analyte standards, that were spiked in the royal jelly samples. Three replicate samples containing all of the analytes in the royal jelly extraction solution as matrix solutions were assayed for each spiked low-, medium-, high-concentration by the standard addition method. The intra-day precision (RSD\%) was found to be less than $13.5 \%$ and the accuracy (recovery\%) ranged from 92.5 to $115.2 \%$ and the inter-day precision (RSD\%) was less than $31.5 \%$ and the accuracy (recovery $\%$ ) ranged from 73.5 to $115.3 \%$. The results, which are shown in
Table S2, indicate that both the repeatability and reproducibility of this method for the quantitation of ATP, ADP, AMP and Ade from royal jelly is satisfactory.

\subsubsection{Matrix effect}

Two types of calibration curve were established for evaluating matrix effects. The calibration curve (1) was prepared by adding 1-10000 ppb mixedstandards to pure water. The other set of calibration curve (2) was prepared by spiking the royal jelly sample with 1-10000 ppb mixed-standards which were subjected to either SPE and dSPE pre-processing or without pre-processing. The differences in slopes were compared to determine the matrix effect using the following relationship: Matrix effect $\%=$ (slope $_{\text {matrix }}-$ slope $\left._{\text {standard }}\right) /$ slope $_{\text {standard }} \times 100 \%$. A value greater than $100 \%$ indicates that the substrate will cause ion-enhancement, while a value less than $100 \%$ indicates that the substrate will lead to ion-suppression. The matrix effect using the slope of the standard calibration curve as the standard was examined by comparing the slope of the matrix effect of the royal jelly samples without pre-processing, the slope of the royal jelly samples with SPE and dSPE pre-processing. As shown in Fig. 3, the matrix effect was significantly reduced by the SPE and dSPE preprocessing methods.

\subsection{Comparison of the applicability between SPE and dSPE method}

In this study, two sample pre-processing methods, SPE and dSPE, were successfully developed to efficiently reduce the matrix effect in the royal jelly samples. The matrix-matched calibration curve, established with the use of the pre-processing methods, was applied for the quantification of nucleotides in complex royal jelly samples. Nevertheless, the use of a matrix-matched calibration curve permits the rapid quantification of nucleotides from royal jelly samples but may also result in inaccurate quantitative results due to different matrix interference levels from each sample. In order to

Table 1. Linearity range, limit of detection (LOD) and limit of quantitation (LOQ) of the method. Parameters were evaluated by 10-point calibration curves in the concentration range of $1-1000 \mathrm{ppb}$ for all analytes $(n=3)$.

\begin{tabular}{llllll}
\hline & Linear equation & Linear range $(\mathrm{ppb})$ & Correlation coefficient $(\mathrm{r})$ & LOD $^{(\mathrm{ppb})^{\mathrm{a}}}$ & LOQ $^{\mathrm{a}}(\mathrm{ppb})^{\mathrm{b}}$ \\
\hline ATP & $\mathrm{Y}=0.00219 \mathrm{X}-0.0185$ & $10-1000$ & 0.9996 & 5 & 10 \\
ADP & $\mathrm{Y}=0.00465 \mathrm{X}-0.0135$ & $5-1000$ & 0.9998 & 2.5 & 5 \\
AMP & $\mathrm{Y}=0.015 \mathrm{X}+0.00867$ & $2.5-1000$ & 0.9999 & 1 & 1 \\
Ade & $\mathrm{Y}=0.00156 \mathrm{X}+0.00407$ & $2.5-1000$ & 0.9995 & 1 & 2.5 \\
\hline
\end{tabular}

\footnotetext{
a $S / N \geq 3$.

b $S / N \geq 10$.
} 
Ade

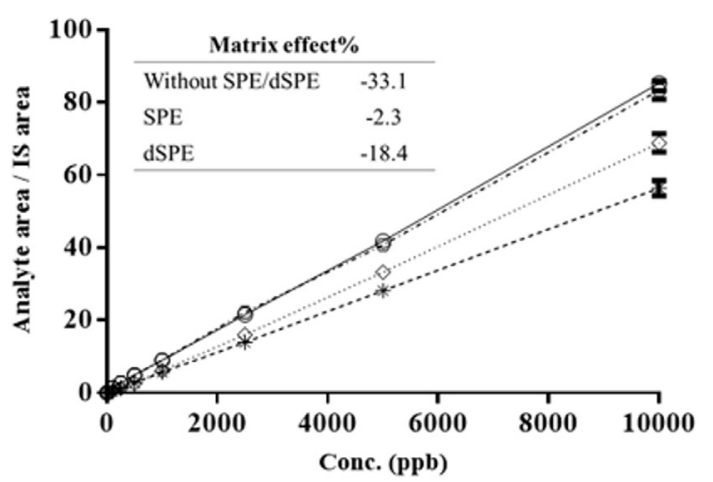

ADP

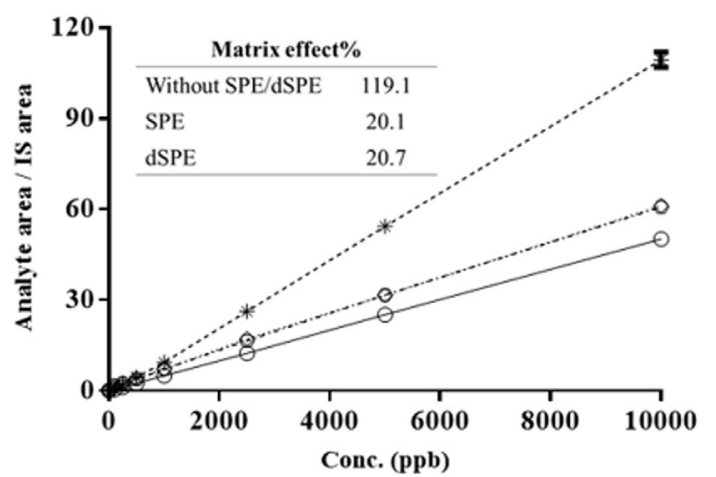

AMP

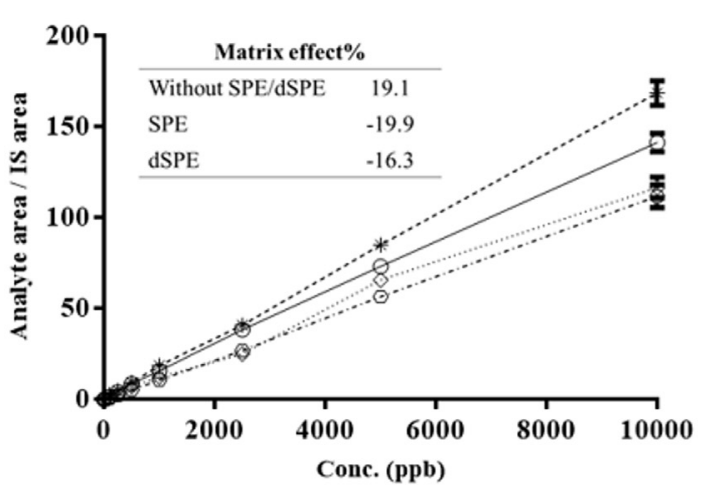

ATP

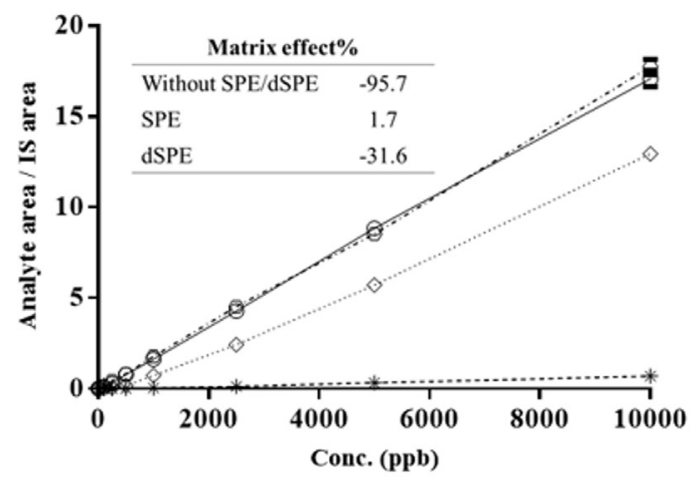

$\odot$ Standard $\quad *$ RJ - Without SPE/dSPE $\leftrightarrow$ RJ - dSPE $\bullet \cdot$ RJ - SPE

Fig. 3. Matrix effects of royal jelly matrix solution without SPE or dSPE, royal jelly matrix solution via SPE and royal jelly matrix solution via dSPE by the slopes of matrix-matched calibration curves. Matrix effect $\%=\left(\right.$ slope $_{\text {matrix }}-$ slope $\left._{\text {standard }}\right) /$ slope $_{\text {standard }} \times 100 \%$.

accurately quantify an analyte in the samples, a quantification calibration curve with the standard addition method was further established by spiking the royal jelly samples with 1-10000 ppb mixedstandards before sample pre-processing. However, solid phase extraction is not suitable for use in the above method due to limitations of the anion exchange capacity of the MAX cartridge. Therefore, a dSPE sample pre-processing method was used for quantification since it is fast, simple, low-cost, and less labor-intensive.

\subsection{Comparison with other methodologies}

In recent years, several methods of nucleotides quantification in royal jelly have appeared in the literature, as illustrated in Table 2. In this study, the appropriate use of dSPE pre-processing procedures can effectively reduce matrix effects. In addition, the proposed LC method not only successfully separated adenine, AMP, ADP and ATP; using MRMMS as a powerful method of detection, resulting in superior sensitivity and better resolution being achieved. Moreover, the used of a cyanopropyl (CN) column proved to be advantageous in terms of a short chromatographic time, satisfactory separation and no need for the use of an organic solvent and a phosphate buffer.

\subsection{Analysis of royal jelly}

In this study, a reliable, sensitive method for the simultaneous quantification of ATP, ADP, AMP and Ade from royal jelly using a dSPE pretreatment and

Table 2. Comparison of this method with other methodologies.

\begin{tabular}{lllllll}
\hline Analysis & Column & Clean-up & LOD & Contents (mg/kg) & Reference \\
\hline UPLC-UV & C18 & SLE & ATP 0.43 mg/kg ADP 0.68 mg/kg AMP 0.42 mg/kg & ATP 51.4 ADP 250.7 AMP 1144.2 & [16] \\
HPLC-UV & C18 & SLE & ATP 46.5 ppb ADP 65.3 ppb AMP 19.5 ppb & ATP 18.7 ADP 154.7 AMP 1506.7 & [17] \\
UPLC-MS/MS Amide & SLE & Ade 59.0 ppb & Ade 9.5 & [32] \\
HPLC-MS/MS CN & SLE-dSPE & ATP 5.0 ppb ADP 2.5 ppb AMP 1.0 ppb Ade 1.0 ppb ATP 11.3 ADP 148.1 AMP 1968.4 Ade 6.5 This study \\
\hline
\end{tabular}


Table 3. Quantification of Ade, AMP, ADP and ATP from 2-day and 3-day royal jelly by dSPE (Standard addition method).

\begin{tabular}{llll}
\hline & Linear equation & Correlation coefficient & Contents $(\mathrm{mg} / \mathrm{kg})$ \\
\hline 3-day royal jelly & & & \\
ATP & $\mathrm{Y}=0.000435 \mathrm{X}+0.0493$ & 0.9990 & 11.3 \\
ADP & $\mathrm{Y}=0.00689 \mathrm{X}+9.96$ & 0.9985 & 144.6 \\
AMP & $\mathrm{Y}=0.00969 \mathrm{X}+206$ & 0.9967 & 2126.0 \\
Ade & $\mathrm{Y}=0.0062 \mathrm{X}+0.0429$ & 0.9994 & 6.9 \\
2-day royal jelly & & & \\
ATP & $\mathrm{Y}=0.0000735 \mathrm{X}+0.00826$ & 0.9975 & 11.2 \\
ADP & $\mathrm{Y}=0.00746 \mathrm{X}+11.3$ & 0.9985 & 151.5 \\
AMP & $\mathrm{Y}=0.0111 \mathrm{X}+201$ & 0.9952 & 1810.8 \\
Ade & $\mathrm{Y}=0.00488 \mathrm{X}+0.033$ & 0.9983 & 6.2 \\
\hline
\end{tabular}

cyanopropyl based LC-MS/MS was developed. This method was further applied to the analysis of royal jelly samples obtained from a local apiary. As shown in Table 3, the levels of ATP could be successfully quantified and their concentrations were found to range from 11.2 to $11.3 \mathrm{mg} / \mathrm{kg}$, the ADP content to range from 111.6 to $151.5 \mathrm{mg} / \mathrm{kg}$, the AMP content to range from 2126.0 to $1810.8 \mathrm{mg} / \mathrm{kg}$ and the Ade content to range from 6.9 to $6.2 \mathrm{mg} / \mathrm{kg}$ in 2-day and 3 -day royal jelly samples.

\section{Conclusions}

In this study, a reliable and sensitive method for the simultaneous quantification of ATP, ADP, AMP and Ade from royal jelly using cyanopropyl based LC-MS/MS was developed. All of the target analytes were successfully separated using a cyanopropyl column combined with quaternary gradient system in less than 9 min, with good resolution and symmetrical peaks. The linear range was $2.5-1000 \mathrm{ng} /$ $\mathrm{mL}$ with correlation coefficient $\mathrm{r}>0.9995$, the LOD of Ade, AMP, ADP and ATP was 1, 1, 2.5 and $5 \mathrm{ng} /$ $\mathrm{mL}$, and the LOQ was 2.5, 2.5, 5 and $10 \mathrm{ng} / \mathrm{mL}$, respectively. Both the precision (RSD $\%<10.5 \%$ ) and accuracy (recovery $81.3-118.4 \%$ ) of the method were satisfactory.
Two pre-processing methods including SLE-SPE and SLE-dSPE were applied to efficiently reduce matrix effects, which were all within the range of $\pm 20.1 \%$ and $\pm 26.4 \%$, respectively. Finally, the dSPE pre-processing method and LC/MS analysis was used for the quantitation of ATP, ADP, AMP and Ade in royal jelly samples. Their concentrations were within $6.2-2126.0 \mathrm{mg} / \mathrm{kg}$, and no significant difference was observed between 2-day and 3-day royal jelly.

Furthermore, ATP from biological samples could degrade to ADP and AMP in a unstable extraction condition. The dSPE method proposed in this article could reduce matrix effect, keep a stable extract environment and be referred by the development of pre-processing method from biological samples in the future.

\section{Acknowledgements}

This research was supported by grants from the Ministry of Science and Technology of Taiwan, Republic of China (MOST 108-2113-M-003-011).

\section{Appendix.}

Table S1. Mass spectrometry parameters for MRM transitions for the analyzed compounds.

\begin{tabular}{|c|c|c|c|c|c|c|c|}
\hline \multirow[t]{2}{*}{ Compound } & \multicolumn{3}{|c|}{ MRM Transition $(\mathrm{m} / \mathrm{z})$} & \multicolumn{4}{|c|}{ CID Voltage (V) } \\
\hline & Precursor Q1 & Product Q3 & Fragmentation & DP & $\mathrm{EP}$ & $\mathrm{CE}$ & CXP \\
\hline \multirow[t]{2}{*}{$\mathrm{AMP}^{15} \mathrm{~N}_{5}(352.1 \mathrm{~g} / \mathrm{mol})$} & 351 & $79^{\mathrm{a}}$ & {$\left[\mathrm{PO}_{3}\right]^{-}$} & -79 & -5 & -40 & -6 \\
\hline & & 97 & {$\left[\mathrm{PO}_{3} \mathrm{H}-\mathrm{OH}\right]^{-}$} & & & -32 & -6 \\
\hline \multirow[t]{2}{*}{ ATP $(507.17 \mathrm{~g} / \mathrm{mol})$} & 506 & $159^{a}$ & {$\left[\left(\mathrm{PO}_{3}\right)_{2} \mathrm{H}\right]^{-}$} & -62 & -8 & -41 & -2 \\
\hline & & 408 & {$\left[\mathrm{C}_{10} \mathrm{H}_{8} \mathrm{~N}_{4} \mathrm{O}_{2} \mathrm{NH}_{2}(\mathrm{OH})_{2}\left(\mathrm{PO}_{3} \mathrm{H}\right)\left(\mathrm{PO}_{2}\right)\right]^{-}$} & & & -30 & -6 \\
\hline \multirow[t]{2}{*}{$\operatorname{ADP}(427.20 \mathrm{~g} / \mathrm{mol})$} & 426 & $134^{\mathrm{a}}$ & {$\left[\mathrm{C}_{5} \mathrm{H}_{4} \mathrm{~N}_{5}\right]^{-}$} & -64 & -11 & -30 & -6 \\
\hline & & 159 & {$\left[\left(\mathrm{PO}_{3}\right)_{2} \mathrm{H}\right]^{-}$} & & & -31 & -3 \\
\hline \multirow[t]{2}{*}{$\operatorname{AMP}(347.20 \mathrm{~g} / \mathrm{mol})$} & 346 & $79^{a}$ & {$\left[\mathrm{PO}_{3}\right]^{-}$} & -84 & -3 & -55 & -3 \\
\hline & & 97 & {$\left[\mathrm{PO}_{3} \mathrm{H}-\mathrm{OH}\right]^{-}$} & & & -30 & -4 \\
\hline \multirow[t]{2}{*}{ Ade $(135.13 \mathrm{~g} / \mathrm{mol})$} & 134 & $107^{\mathrm{a}}$ & {$\left[\mathrm{C}_{4} \mathrm{H}_{3} \mathrm{~N}_{4}\right]^{-}$} & -81 & -8 & -24 & -6 \\
\hline & & 65 & {$\left[\mathrm{C}_{3} \mathrm{HN}_{2}\right]^{-}$} & & & -40 & -2 \\
\hline
\end{tabular}

\footnotetext{
${ }^{\text {a }}$ MRM transition for quantitative analysis.
} 
Table S2. Intra-day and Inter-day precision and recovery at low-, medium-, high-concentrations of analyte standards, that were spiked in royal jelly sample $(n=3)$.

\begin{tabular}{llllll}
\hline Intra-day $(\mathrm{n}=3)$ & & & & & \\
\hline Spiked $(\mu \mathrm{g} / \mathrm{mL})$ & & ATP & ADP & AMP & Ade \\
\hline 0.5 & Recovery\% & 115.2 & 91.7 & 108.5 & 102.6 \\
& RSD\% & 4.5 & 13.5 & 8.5 & 14.7 \\
1 & Recovery\% & 100.2 & 99.2 & 109.3 & 92.5 \\
& RSD\% & 5.4 & 15.5 & 6.3 & 14.5 \\
5 & Recovery\% & 111.0 & 112.0 & 101.4 & 93.7 \\
& RSD\% & 6.8 & 10.4 & 14.8 & 3.3 \\
\hline Inter-day $(\mathbf{n}=\mathbf{9 )}$ & & & & & \\
\hline Spiked $(\mu \mathrm{g} / \mathrm{mL})$ & & ATP & ADP & AMP & Ade \\
\hline $\mathbf{0 . 5}$ & Recovery\% & 97.1 & 101.1 & 112.5 & 97.3 \\
& RSD\% & 19.5 & 12.2 & 4.8 & 15.3 \\
$\mathbf{1}$ & Recovery\% & 73.5 & 110.1 & 111.1 & 99.7 \\
& RSD\% & 31.5 & 8.6 & 4.5 & 16.2 \\
5 & Recovery\% & 87.3 & 115.3 & 96.0 & 83.8 \\
& RSD\% & 26.1 & 3.0 & 4.9 & 17.5 \\
\hline
\end{tabular}

\section{References}

[1] Langen P, Hucho F. Karl lohmann and the discovery of ATP. Angew Chem Int Ed 2008;47:1824-7.

[2] Hardie DG, Hawley SA. AMP-activated protein kinase: the energy charge hypothesis revisited. Bioessays 2001;23: 1112-9.

[3] Domínguez-Álvarez J, Mateos-Vivas M, RodríguezGonzalo E, García-Gómez D, Bustamante-Rangel M, Zamarreño M-MD, et al. Determination of nucleosides and nucleotides in food samples by using liquid chromatography and capillary electrophoresis. TrAC Trends Anal Chem (Reference Ed) 2017;92:12-31.

[4] Verkerk R. Nucleotides: speculation on lifestyle-induced essentiality. NHD Clinical 2011;64:29-32.

[5] Takenaka T, Echigo T. Chemical composition of royal jelly. Bulletin of the Faculty of Agriculture. Tamagawa University; 1980. p. 71-8.

[6] Echigo T. Comparative studies on chemical composition of honey, royal jelly and pollen loads. Bull Fac Agric Tamagawa Univ 1986;26:1-12.

[7] Guo H, Kouzuma Y, Yonekura M. Structures and properties of antioxidative peptides derived from royal jelly protein. Food Chem 2009;113:238-45.

[8] Bloodworth BC, Harn CS, Hock CT, Boon YO. Liquid chromatographic determination of trans-10-hydroxy-2-decenoic acid content of commercial products containing royal jelly. J AOAC Int 1995;78:1019-23.

[9] Han B, Li C, Zhang L, Fang Y, Feng M, Li J. Novel royal jelly proteins identified by gel-based and gel-free proteomics. J Agric Food Chem 2011;59:10346-55.

[10] Zamani Z, Reisi P, Alaei H, Pilehvarian AA. Effect of Royal Jelly on spatial learning and memory in rat model of streptozotocin-induced sporadic Alzheimer's disease. Adv Biomed Res 2012;1.

[11] Honda Y, Fujita Y, Maruyama H, Araki Y, Ichihara K, Sato A, et al. Lifespan-extending effects of royal jelly and its related substances on the nematode Caenorhabditis elegans. PloS One 2011;6.

[12] Kim J, Kim Y, Yun H, Park H, Kim SY, Lee K-G, et al. Royal jelly enhances migration of human dermal fibroblasts and alters the levels of cholesterol and sphinganine in an in vitro wound healing model. Nutr. Res. Pract. 2010;4:362-8.
[13] Park HM, Hwang E, Lee KG, Han S-M, Cho Y, Kim SY. Royal jelly protects against ultraviolet $\mathrm{B}$-induced photoaging in human skin fibroblasts via enhancing collagen production. J Med Food 2011;14:899-906.

[14] Siavash M, Shokri S, Haghighi S, Shahtalebi MA, Farajzadehgan Z. The efficacy of topical royal jelly on healing of diabetic foot ulcers: a double-blind placebo-controlled clinical trial. Int Wound J 2015;12:137-42.

[15] Pasupuleti VR, Sammugam L, Ramesh N, Gan SH. Honey, propolis, and royal jelly: a comprehensive review of their biological actions and health benefits. Oxid. Med. Cell. Longev. 2017;2017.

[16] Zhou L, Xue X, Zhou J, Li Y, Zhao J, Wu L. Fast determination of adenosine $5^{\prime}$-triphosphate (ATP) and its catabolites in royal jelly using ultraperformance liquid chromatography. J Agric Food Chem 2012;60:8994-9.

[17] Wu L, Chen L, Selvaraj JN, Wei Y, Wang Y, Li Y, et al. Identification of the distribution of adenosine phosphates, nucleosides and nucleobases in royal jelly. Food Chem 2015; 173:1111-8.

[18] Wei Y, Chen Y, Li H, Shuang S, Dong C, Wang G. An exonuclease I-based label-free fluorometric aptasensor for adenosine triphosphate (ATP) detection with a wide concentration range. Biosens Bioelectron 2015;63:311-6.

[19] Zhu P, Wang S, Wang J, Zhou L, Shi P. A capillary zone electrophoresis method for adenine nucleotides analysis in Saccharomyces cerevisiae. J Chromatogr B 2016;1008:156-63.

[20] Liu J-X, Aerts JT, Rubakhin SS, Zhang X-X, Sweedler JV. Analysis of endogenous nucleotides by single cell capillary electrophoresis-mass spectrometry. Analyst 2014;139: 5835-42.

[21] Zinellu A, Pasciu V, Sotgia S, Scanu B, Berlinguer F, Leoni G, et al. Capillary electrophoresis with laser-induced fluorescence detection for ATP quantification in spermatozoa and oocytes. Anal Bioanal Chem 2010;398:2109-16.

[22] Contreras-Sanz A, Scott-Ward TS, Gill HS, Jacoby JC, Birch RE, Malone-Lee J, et al. Simultaneous quantification of 12 different nucleotides and nucleosides released from renal epithelium and in human urine samples using ion-pair reversed-phase HPLC. Purinergic Signal 2012;8:741-51.

[23] Magdenoska O, Martinussen J, Thykaer J, Nielsen KF. Dispersive solid phase extraction combined with ion-pair ultra high-performance liquid chromatography tandem mass spectrometry for quantification of nucleotides in Lactococcus lactis. Anal Biochem 2013;440:166-77.

[24] Jia J, Zhang H, Zhao L, Zhu Z-y, Zhang G-q, Chai Y-f. An optimized Ion-Pair HPLC method for simultaneous analysis of nucleoside triphosphate levels in hepatoma cell line. Chromatographia 2011;73:755-9.

[25] Xue X, Wang F, Zhou J, Chen F, Li Y, Zhao J. Online cleanup of accelerated solvent extractions for determination of adenosine $5^{\prime}$-triphosphate (ATP), adenosine $5^{\prime}$-diphosphate (ADP), and adenosine $5^{\prime}$-monophosphate (AMP) in royal jelly using high-performance liquid chromatography. J Agric Food Chem 2009;57:4500-5.

[26] Gill BD, Indyk HE, Manley-Harris M. Analysis of nucleosides and nucleotides in infant formula by liquid chromatography-tandem mass spectrometry. Anal Bioanal Chem 2013;405:5311-9.

[27] Mateos-Vivas M, Rodríguez-Gonzalo E, DomínguezÁlvarez J, García-Gómez D, Carabias-Martínez R. Determination of nucleosides and nucleotides in baby foods by hydrophilic interaction chromatography coupled to tandem mass spectrometry in the presence of hydrophilic ion-pairing reagents. Food Chem 2016;211:827-35.

[28] Mora L, Hernández-Cázares AS, Aristoy M-C, Toldrá F. Hydrophilic interaction chromatographic determination of adenosine triphosphate and its metabolites. Food Chem 2010;123:1282-8.

[29] Inoue K, Dowell D. HILIC-MS/MS method for the quantitation of nucleotides in infant formula and adult nutritional formula: first Action 2011.21. J AOAC Int 2012;95:603-5. 
[30] Marrubini G, Appelblad P, Maietta M, Papetti A. Hydrophilic interaction chromatography in food matrices analysis: an updated review. Food Chem 2018;257:53-66.

[31] Zhu B, Wei H, Wang Q, Li F, Dai J, Yan C, et al. A simultaneously quantitative method to profiling twenty endogenous nucleosides and nucleotides in cancer cells using UHPLC-MS/MS. Talanta 2018;179:615-23.
[32] Pina A, Begou O, Kanelis D, Gika H, Kalogiannis S, Tananaki C, et al. Targeted profiling of hydrophilic constituents of royal jelly by hydrophilic interaction liquid chromatography-tandem mass spectrometry. J Chromatogr 2018;1531:53-63. 